\title{
The impact of smoking on failure rates, postoperative infection and marginal bone loss of dental implants
}

\author{
Abstracted from \\ Chrcanovic BR, Albrektsson T, Wennerberg A. \\ Smoking and dental implants: A systematic review and meta-analysis. \\ J Dent. 2015; 43: 487-498. doi: 10.1016/j.jdent.2015.03.003. Epub 2015 Mar 14 \\ Address for correspondence: BR Chrcanovic, Department of Prosthodontics, \\ Faculty of Odontology, Malmö University, Malmö, Sweden. \\ E-mail: Bruno.chrcanovic@mah.se; brunochrcanovic@hotmail.com
}

Question: In patients undergoing implant placement, are patients who smoke compared with those who do not at higher risk for implant failure, postoperative infection and greater marginal bone loss?

Data sources Electronic searches were undertaken in PubMed/ Medline, Web of Science, Cochrane Oral Health Group Trials Register as well as hand searching.

Study selection Clinical human studies, either randomised or not, providing outcome data for dental implant failure in smokers and nonsmokers in any group of patients with no follow-up restrictions. Patients smoking a minimum of one cigarette a day were classified as smokers and implant failure was considered as the complete loss of the implant. Data extraction and synthesis Three authors independently participated in the inclusion criteria and disagreements were resolved by discussion. Quality assessment of the studies was performed using a scale to appraise observational studies. From the selected studies, data were extracted when available which included year of publication, study design, country, setting, number of patients, type of smokers, age, follow-up, days of antibiotic prophylaxis, failure, postoperative infection, marginal bone loss, implant surface, dental arch receiving implants, type of prosthetic rehabilitation and opposing dentition. For the metaanalysis, implant failure and postoperative infection were evaluated as dichotomous outcomes, while weighted mean differences were used for marginal bone loss as a continuous outcome. The statistical unit was the implant. Heterogeneity was calculated using $1^{2}$ test.

Results From 1432 records identified, 107 publications were included in the review for the qualitative and quantitative synthesis. One hundred and four studies compared implant failure rates. From the total studies, the authors judged 85 to be of high quality and 22 of moderate quality. The meta-analysis for failure rate resulted in a statistically significant overall result of risk ratio (RR) of 2.23 (95\% Cl 1.96-2.53) heterogeneity of $\mathrm{I}^{2}: 51 \%$. The meta-analysis of four studies evaluating the risk of postoperative infections in smokers presented a statistically significant result with an RR: $2.01,95 \% \mathrm{Cl} 1.09-3.72$, heterogeneity $\mathrm{I}^{2}: 0 \%$, and the marginal bone loss had an overall statistically significant difference of MD $0.32,95 \% \mathrm{Cl} 0,21-043$; heterogeneity in this case was $1^{2}: 95 \%$.

Conclusions The insertion of implants in smokers affected the failure rates, the risk of postoperative infections, as well as the marginal bone loss. The results should be interpreted with caution due to the presence of uncontrolled confounding factors in the included studies.

\section{Commentary}

Several reviews have been performed on the topic of implant failure in smokers compared to non-smokers. ${ }^{1-6}$

Problems are due to the effects of tobacco and nicotine on the oral soft and hard tissues. Hence, the impact of smoking and the failure of dental implants has been a constant topic for discussion for the last decade or longer.

This review proposed the question of the impact of smoking compared to not smoking and the higher risk of implant failure rate, postoperative infection and greater marginal bone loss (MBL).

To suitably conduct the review the authors followed the PRISMA guidelines to perform a systematic review. A search was performed in several databases. Inclusion criteria were set to human clinical studies with no time or language restrictions for the publication.

Interestingly, the authors considered smoking one cigarette a day was 'smoking' and implant failure was represented as complete loss of the implant. That is probably the reason that the final study selection included many more studies than other reviews: (107 studies); 71 retrospective studies, 16 prospective studies, 16 controlled clinical trials (CCT) and four randomised clinical trials (RCTs). The review reported that seven of the CCTs and the four RCTs included in the studies were not controlled for the smoking habit.

The quality of the studies was assessed using a specific tool for critical appraisal of non-randomised clinical trials: the NewcastleOttawa scale (NOS).

Data from the studies were extracted by only one reviewer. Ordinarily this task uses more than one author to reduce bias with disagreements, being resolved by a third reviewer.

The unit analysed in the review was the 'implant', and the 107 studies collected data from 19,836 implants in smokers $(1,259$ failures) and 60,464 implants in non-smokers (1,923 failures), and calculated the risk ratio( RR) for implant failure as 2.23 (95\% CI 1.96-2.53). In other words, the data for the review indicate that smoking doubles the risk of implant failure. However, the risk of implant failure, postoperative infections and marginal bone loss are dependent upon several conditions and risk factors from the site (such as the patient's medical condition, bone, remaining dentition, parafunctional habits, operator expertise, materials used and implant type).

Based on this review, we can say that there is an increased chance of implant failure in smokers. However, the applicability and the interpretation of the results (even when similar to other reviews) 
should not be held with complete confidence. Bias may be in the final results due to the limitations in the methodology of the included studies.

James R Keenan and Analia Veitz-Keenan New York College of Dentistry, New York, USA

1. Moraschini V, Barboza Ed. Success of dental implants in smokers and non-smokers: a systematic review and meta-analysis. Int J Oral Maxillofac Surg 2016; 45: 205215. doi: 10.1016/j.ijom.2015.08.996. Epub 2015 Sep 15. Review. PubMed PMID: 26385308.

2. Chen H, Liu N, Xu X, Qu X, Lu E. Smoking, radiotherapy, diabetes and osteoporosis as risk factors for dental implant failure: a meta-analysis. PLoS One 2013; 8: e71955. doi: 10.1371/journal.pone.0071955. Print 2013. PubMed PMID: 23940794; PubMed Central PMCID: PMC3733795.

3. Chambrone L, Preshaw PM, Ferreira JD, Rodrigues JA, Cassoni A, Shibli JA. Effects of tobacco smoking on the survival rate of dental implants placed in areas of maxillary sinus floor augmentation: a systematic review. Clin Oral Implants Res 2014; 25: 408416. doi: 10.1111/clr.12186. Epub 2013 May 7. Review. PubMed PMID: 23651315.

4. Klokkevold PR, Han TJ. How do smoking, diabetes, and periodontitis affect outcomes of implant treatment? Int J Oral Maxillofac Implants 2007; 22 Suppl: 173-202. Review. Erratum in: Int / Oral Maxillofac Implants 2008; 23: 56. PubMed PMID: 18437796.

5. Strietzel FP, Reichart PA, Kale A, Kulkarni M, Wegner B, Küchler I. Smoking interferes with the prognosis of dental implant treatment: a systematic review and meta-

analysis. / Clin Periodontol 2007; 34: 523-544. Review. PubMed PMID: 17509093.

6. Hinode D, Tanabe S, Yokoyama M, Fujisawa K, Yamauchi E, Miyamoto Y. Influence of smoking on osseointegrated implant failure: a meta-analysis. Clin Oral Implants Res 2006; 17: 473-48. Review. PubMed PMID: 16907781.

Evidence-Based Dentistry (2016) 17, 4-5. doi:10.1038/sj.ebd.6401144 